\title{
A comparison of the duration of post-treatment protection of artemether-lumefantrine, dihydroartemisinin-piperaquine and artesunate-amodiaquine for the treatment of uncomplicated malaria
}

\author{
Michael T Bretscher ${ }^{1 *}$, Jamie T Griffin ${ }^{1}$, Pierre Hugo ${ }^{2}$, Mark Baker ${ }^{2}$, Azra Ghani ${ }^{1}$, Lucy Okell ${ }^{1}$, \\ The AS-AQ Post-Treatment Prophylaxis Study Group ${ }^{1}$
}

From Challanges in malaria research: Core science and innovation

Oxford, UK. 22-24 September 2014

\section{Background}

Five artemisinin combination therapies (ACTs) are currently recommended for treatment of uncomplicated malaria in Africa. Whilst the artemisinin component has a short half-life, the partner drugs give rise to differing durations of post-treatment prophylaxis, protecting from re-infection and reducing transmission. We compared the duration of post-treatment prophylaxis of artemetherlumefantrine (AL), dihydroartemisinin-piperaquine (DHA$\mathrm{PPQ}$ ) and artemisinine-amodiaquine (AS-AQ).

\section{Materials and methods}

We pooled the results from analyses of 6 trials (1651 individuals) of AL versus DHA-PPQ and from 18 trials (5060 individuals) of $\mathrm{AL}$ versus $\mathrm{AS}-\mathrm{AQ}$. The time to re-infection after treatment was used to estimate the duration of posttreatment protection accounting for the variation in transmission intensity between settings. This duration was then used in a mathematical model of malaria transmission to estimate the comparative public health impact of each drug used for 1st-line treatment in low, medium and high transmission settings.

\section{Results}

We estimated a mean duration of post-treatment protection of 10.7 days (95\% CI: 10.2-11.2) and 13.8 days (95\% CI: 10.2-22.8) for AL from the two analyses, 29.4 days
(95\% CI: 16.4-48.8) for DHA-PPQ, and 11.8 days (95\% CI: 11.0-12.5) for ASAQ. There was no substantial difference in the public health impact of using $A L$ versus $A S-A Q$. Use of DHA-PPQ for 1st-line treatment averted 8.3 additional cases per 1,000 people $(0.8 \%$ of all cases) over 5 years compared to AL or AS-AQ. This protective effect was stronger in areas of high transmission as well as in seasonal settings.

\section{Conclusions}

While it is important to maintain a diverse set of first-line treatments for uncomplicated malaria, our results suggest that partner drugs with a longer half-life could be beneficial in reducing repeat episodes and onward transmission, particularly in high and seasonal transmission areas.

\section{Authors' details \\ 'Imperial College London, London, UK. ${ }^{2}$ Medicines for Malaria Venture} (MMV), Geneva, Switzerland.

Published: 22 September 2014

doi:10.1186/1475-2875-13-S1-P19

Cite this article as: Bretscher et al: A comparison of the duration of post-treatment protection of artemether-lumefantrine,

dihydroartemisinin-piperaquine and artesunate-amodiaquine for the treatment of uncomplicated malaria. Malaria Journal 2014 13(Suppl 1): P19.

${ }^{1}$ Imperial College London, London, UK

Full list of author information is available at the end of the article 